\title{
Youngest agamid lizards from Western Europe (Sierra de Quibas, Spain, late Early Pleistocene)
}

Hugues-Alexandre Blain, Salvador Bailon, Jordi Agustí, Pedro Piñero-García, Iván Lozano-Fernández, Paloma Sevilla, Juan Manuel López-García, Gregorio Romero, and Miguel Angel Mancheño Acta Palaeontologica Polonica 59 (4), 2014: 873-878 doi: http://dx.doi.org/10.4202/app.2012.0141

Here we report a new Early Pleistocene record of agamid lizards (Agamidae indet.) from the Spanish locality of Quibas (ca. 1.3-1.0 Ma), which represents the latest evidence of the family in Western Europe. Up to now, the family Agamidae was considered to have disappeared in this region at 1.8 Ma. This new record implies the survival of agamid lizards for a large part of the Early Pleistocene in the southernmost Iberian Peninsula, probably because of favourable climatic and environmental conditions. Consequently, the formerly detected Pliocene / Pleistocene boundary is no longer recognisable in the evolution of the herpetological assemblages in Western Europe.

Hugues-Alexandre Blain [hablain@iphes.cat], Pedro Piñero-García [ppinero@iphes.cat ], Iván Lozano-Fernández [ilozano@iphes.cat], IPHES, Institut Català de Paleoecologia Humana i Evolució Social, c/ Escorxador s/n, 43003 Tarragona, Spain and Àrea de Prehistòria, Universitat Rovira i Virgili (URV), Avinguda de Catalunya 35, 43002 Tarragona, Spain; Salvador Bailon [salvador.bailon@mnhn.fr ], UMR 7149-7209-USM 303 “Archéozoologie et Histoire des Sociétés”, Département EGB, Muséum national d'Histoire naturelle, Bâtiment d'Anatomie Comparée CP55, 55 rue Buffon, 75005 Paris, France; Jordi Agustí [jordi.agusti@icrea.cat ], ICREA, Institució Catalana de Recerca i Estudis Avançats, Barcelona, Spain; IPHES, Institut Català de Paleoecologia Humana i Evolució Social, c/ Escorxador s/n, 43003 Tarragona, Spain; and Àrea de Prehistòria, Universitat Rovira i Virgili (URV), Avinguda de Catalunya 35, 43002 Tarragona, Spain; Paloma Sevilla [psevilla@geo.ucm.es ], Departamento de Paleontología, Facultad de Ciencias Geológicas (UCM) C/ Jose Antonio Novais 2, 28040 Madrid, Spain; Juan Manuel López-García [1pzjmn@unife.it], Gruppo di Ricerca di Paleobiologia e Preistoria, Dipartimento di Studi Umanistiche, Università degli Studi di Ferrara, C.so Ercole I d’Este 32, 44121 Ferrara, Italy; Gregorio Romero Sánchez [gromero@um.es], Dpto. Química Agrícola, Geología y Edafología, Facultad de Química, Universidad de Murcia, Campus de Espinardo, 30100 Murcia, Spain and Servicio de Patrimonio Histórico, Dirección General de Bienes Culturales, Consejería de Cultura y Turismo, Plaza Julián Romea 4, 30071 Murcia, Spain; Miguel Angel Mancheño [cheno@um.es], Dpto. Química Agrícola, Geología y Edafología, Facultad de 
Química, Universidad de Murcia, Campus de Espinardo, 30100 Murcia, Spain.

This is an open-access article distributed under the terms of the Creative Commons Attribution License (for details please see creativecommons.org), which permits unrestricted use, distribution, and reproduction in any medium, provided the original author and source are credited.

Fofill Full text $(393.9 \mathrm{kB})$ 